\title{
Spatial coating inhomogeneity of highly reflective mirrors determined by cavity ringdown measurements
}

\author{
Zhongqi Tan, ${ }^{1,3}$ Kaiyong Yang, ${ }^{1}$ Xingwu Long, ${ }^{1}$ Yibo Zhang, ${ }^{1}$ and Hans-Peter Loock ${ }^{2,4}$ \\ ${ }^{1}$ College of Optoelectronic Science \& Engineering, National University of Defense Technology, Changsha 410073, China \\ ${ }^{2}$ Department of Chemistry, Queen's University, Kingston, ON, K7L 3N6, Canada \\ ${ }^{3}$ e-mail: zhqitan@sina.com \\ ${ }^{4}$ e-mail: hploock@chem.queensu.ca
}

Received 15 November 2013; revised 27 March 2014; accepted 28 March 2014; posted 28 March 2014 (Doc. ID 201237); published 30 April 2014

\begin{abstract}
The inhomogeneity of high-reflectivity mirror coatings is a potential error source in the application of the cavity ringdown technique. Here, the ringdown times for different transverse modes were recorded. Together with the observed spatial distribution of these modes the ringdown times can be used to approximately locate the position of coating defects. A simple model based on a weighted sum of HermiteGaussian mode functions is used to explain the experimental results. (C) 2014 Optical Society of America OCIS codes: (310.0310) Thin films; (120.0120) Instrumentation, measurement, and metrology; (310.6860) Thin films, optical properties; (120.5700) Reflection.

http://dx.doi.org/10.1364/AO.53.002917
\end{abstract}

\section{Introduction}

The cavity ringdown (CRD) technique has been widely applied in trace gas spectroscopy [1], spectroscopy of very weak optical transitions [2], as well as the characterization of low-loss optical coatings, including high-reflection and antireflection coatings [3-6]. Several commercial spectrometers are based on the CRD technique and are sold for, e.g., trace gas monitoring purposes and as research tools. Compared to other high-sensitivity spectroscopic techniques, the CRD technique has a number of advantages, such as a rather simple and straightforward experimental setup, the intrinsic insensitivity to light source intensity fluctuations, and the capability of measuring attenuation on an absolute scale [7-10].

In $C \bar{R} \bar{D}$ spectroscopy, the accurate determination of cavity loss is based on the decay time measurement

$1559-128 X / 14 / 132917-07 \$ 15.00 / 0$

(C) 2014 Optical Society of America of pulsed or continuous-wave laser light in a low-loss optical cavity [11-14]. In past studies the limits of the CRD technique have been discussed by many authors, especially with regards to the accuracy of the determination of the decay time. It appears to be commonly accepted that cavities that are locked to a single cavity mode provide the highest accuracies for the optical loss measurements [14-16]. Simultaneous excitation of multiple cavity modes is known to cause beating and with it a reduced CRD time accuracy [13]. The many transverse modes that can be found near a longitudinal mode resonance have been observed to interconvert $[17,18]$ and may generally exhibit different ringdown times in addition to slightly different resonant frequencies [13]. Aside from different resonant frequencies and optical losses the different transverse and longitudinal cavity modes typically also have different coupling efficiencies to the incoming laser modes.

Of particular importance to robust CRD measurements is the spatial uniformity of the reflectivity of the two (or more) mirrors. Since every transverse 
mode samples a different area on the mirrors, the differences of their respective ringdown times can be associated with a spatial reflectivity distribution function, which provides a measure for the inhomogeneity of the mirror coatings. Conversely, one can use the differences in optical loss associated with the respective transverse cavity modes to create a "reflectivity map" of the mirrors-keeping in mind that the contributions from the two mirrors cannot be easily separated. Although of large historical importance $[3,19]$, the coating inhomogeneity of high-reflectivity mirrors has rarely been investigated, aside from several early studies [4] including the first application of phase-shift CRD to the characterization of mirror coatings by Herbelin et al. [3] .

In this report, we examine the influence of coating inhomogeneity on the CRD measurement. In particular, we use the ringdown times of different transverse cavity modes to create a "reflectivity map" for the mirrors. We show that one can synthesize a reflectivity map from a sum of $\mathrm{TEM}_{m, n}$ basis functions or from the spatial intensity distributions associated with each experimentally obtained transverse cavity mode. This may be regarded as an analogy to the synthesis of a waveform using a sum of weighted Fourier components.

\section{Theory}

The transverse mode pattern of cavity modes in a two-mirror cavity can be described either in a Cartesian coordinate system or in a cylindrical coordinate system. In the former case Hermite-Gaussian transverse mode patterns and their linear combinations give an adequate representation of the intensity distribution inside a cavity. The normalized Hermite-Gaussian functions of order $m$ and $n$ can be described as [20-22]

$$
\begin{aligned}
\mathrm{HG}_{m}(x)= & \left(\frac{2}{\pi w^{2}}\right)^{1 / 4} \frac{1}{\sqrt{2^{m} m !}} H_{m}\left(\frac{\sqrt{2} x}{w}\right) \exp \left(\frac{-x^{2}}{w^{2}}\right) \\
& m=0,1,2,3, \ldots \\
\mathrm{HG}_{n}(y)= & \left(\frac{2}{\pi w^{2}}\right)^{1 / 4} \frac{1}{\sqrt{2^{n} n !}} H_{n}\left(\frac{\sqrt{2} y}{w}\right) \exp \left(\frac{-y^{2}}{w^{2}}\right) \\
& n=0,1,2,3, \ldots,
\end{aligned}
$$

where $H_{m}(x)$ and $H_{n}(y)$ are Hermite polynomial functions of order $m$ and $n$, respectively, and $w$ is the beam waist of the lowest order mode. The intensity distribution of each $\mathrm{TEM}_{m, n}$ mode can be calculated from the Hermite-Gaussian basis functions

$$
I_{m, n}(x, y)=I_{m, n}^{0}\left[\mathrm{HG}_{m}(x)\right]^{2}\left[\mathrm{HG}_{n}(y)\right]^{2} .
$$

The normalization factors in Eq. (1) assure that for each mode

$$
1=\int_{x=-\infty}^{\infty} \operatorname{HG}(x)^{2} \mathrm{~d} x=\int_{y=-\infty}^{\infty} \operatorname{HG}(y)^{2} \mathrm{~d} y .
$$

And therefore,

$$
I^{0}=I_{m, n}^{0}=\int_{x, y=-\infty}^{\infty} I_{m, n}(x, y) \mathrm{d} x \mathrm{~d} y .
$$

In the analysis of the experimental data we scale the spatially integrated intensity of each mode to the same value, $I^{0}$. Examples of calculated $I_{m, n}(x, y)$ functions are given in Fig. 1 .

We intend to obtain information about the mirror coating's homogeneity from a "reflectivity map." The transverse modes on each of the mirrors can be added incoherently and in the limiting case of a very large number of transverse modes the sum of all their intensities covers the mirror surface smoothly with a maximum on the cavity axis:

$$
\bar{I}(x, y)=\frac{\sum_{n, m=0}^{N, M} I_{m, n}(x, y)}{(N+1)(M+1)} .
$$

The size of the area that can be interrogated in this manner depends on the size of the highest order HG-mode, which is about $4 w^{2}(N+1)^{1 / 2}(M+1)^{1 / 2}$ [22]. It is well-known that Hermite-Gaussian functions form a complete basis set [22], and the interrogation area as well as the spatial resolution can be increased by including more modes in the analysis.

In a practical application the number of available mode functions is finite. We used $N=M=6$ for 49 TEM modes in the simulation and a total of 13 or 15 modes in the present experiments. As indicated in Eq. (4), we give each mode the same weight.

When the mirror surface exhibits defects in the coating, the reflectivity of each mode is reduced. Figure 2(A) shows the location of a simulated defect as the product, $\bar{I}(x, y) \cdot R(x, y)$, which is equivalent to

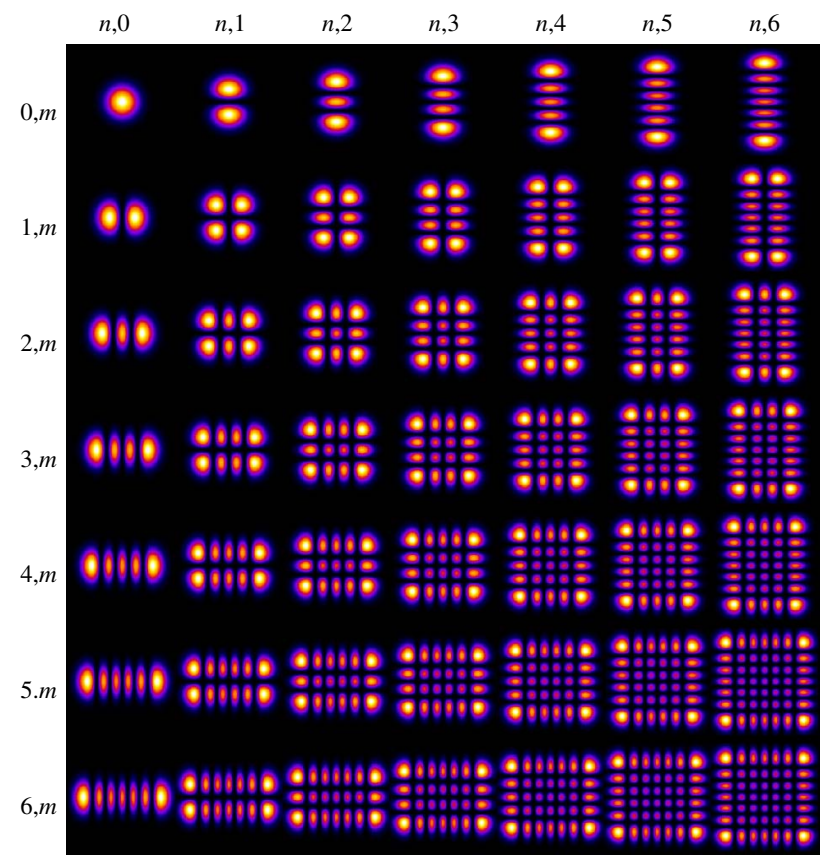

Fig. 1. Simulated intensity distribution functions for a set of Hermite-Gaussian transverse cavity modes. 

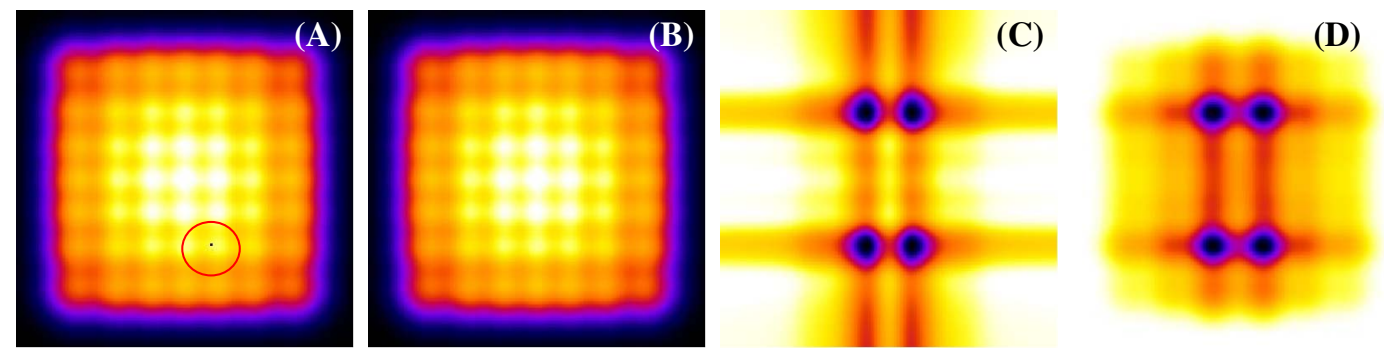

Fig. 2. Intensity distribution function assuming an incoherent sum of all transverse mode intensities functions. The sum includes all Hermite-Gaussian basis functions of Fig. 1 with $n, m=0$ to 6. (A) All 49 simulated Hermite-Gaussian functions each contain a small defect, $\bar{I}(x, y) \circ R(x, y)$. The defect is in the center of the red circle. (B) Sum of all 49 simulated Hermite-Gaussian functions without a defect but weighted according to the fraction by which each mode samples the defect, $\sum_{n, m=0}^{N, M} c_{m, n} I_{m, n}(x, y)$. The differences to (A) are barely visible. (C) Ratio between (A) and (B) showing the simulated reconstruction of the defect location, $R^{\prime}(x, y)$, as described in Eq. (7). The comparison with (A) shows that all modes sampling the defect (in the lower right maximum) are attenuated. (D) Difference between (A) and (B) showing the simulated reconstruction of the defect location, $R^{\prime \prime}(x, y)$, as described in Eq. (10).

a Hadamard product $\bar{I}(x, y) \circ R(x, y)$ if the image is discretized into pixels.

For each TEM $_{m, n}$ mode we define a weighting factor that is calculated from the Hadamard product of the reflectivity map, $R(x, y)$ and the mode's intensity distribution:

$$
c_{m, n}=\frac{1}{I_{0}} \int_{x, y=-\infty}^{\infty} I_{m, n}(x, y) \circ R(x, y) \mathrm{d} x \mathrm{~d} y .
$$

This weighting factor is close to unity, if the mode has intensity nodes at the defect sites, and it can be substantially less than unity when an intensity maximum falls onto a defect site.

If the weighting factors have been obtained experimentally, e.g., by using the ringdown times for each mode, one can obtain an approximation of the reflectivity map $R^{\prime}(x, y)$ [Fig. 2(C)] from the ratio of the sum of weighted transverse mode functions [Fig. 2(B)] to the finite sum of all available transverse modes [Eq. (5)] [Fig. 2(A)]. We define this reconstructed reflectivity map as

$$
\begin{aligned}
R^{\prime}(x, y) & =\frac{\sum_{n, m=0}^{N, M} c_{m, n} I_{m, n}(x, y)}{\bar{I}(x, y)(N+1)(M+1)} \\
& =\frac{\sum_{n, m=0}^{N, M} c_{m, n} I_{m, n}(x, y)}{\sum_{n, m=0}^{N, M} I_{m, n}(x, y)} .
\end{aligned}
$$

Even if it was possible to record the weighting factor, $c_{m, n}$, and spatial distribution, $I_{m, n}(x, y)$, of a very large number of modes, the reconstructed reflectivity map $R^{\prime}(x, y)$ cannot be identical to the actual reflectivity map, $R(x, y)$, since the Hermite-Gaussian modes all exhibit inversion symmetry and two symmetry planes. This prevents the experimenter from identifying the quadrant in which the defect is located. Without including modes that break the symmetry, the analysis can therefore provide no more than an estimate of the inhomogeneity and the reconstructed reflectivity map $R^{\prime}(x, y)$ cannot be identical to $R(x, y)$. In practice, transverse modes are not restricted exactly by the symmetry relationships (see below) and the symmetry restrictions then no longer apply.

Of course, the accuracy and resolution of the reconstructed reflectivity map depends on the type of basis functions and on their number $(N+1) \times(M+1)$ that are included in the sum. The spatial resolution of Hermite-Gaussian type modes is ultimately limited by the distance between nodes in $I_{M . N}(x, y)$, which may be approximated as $\delta x=2 w / M^{1 / 2}$ and $\delta y=2 w / N^{1 / 2}[22]$.

In our experiment the optical loss of each mode is calculated from the measurement of the CRD time, $\tau_{n, m}$,

$$
\Gamma_{n, m}=1-\exp \left(-\frac{t_{\mathrm{RT}}}{\tau_{n, m}}\right)
$$

where the round trip time $t_{\mathrm{RT}}=2 L / c_{0}=4 \mathrm{~ns}$ for our $60 \mathrm{~cm}$ cavity. The optical loss due to mirror contamination is

$$
\Delta \Gamma_{n, m}=\exp \left(-\frac{t_{\mathrm{RT}}}{\tau^{0}}\right)-\exp \left(-\frac{t_{\mathrm{RT}}}{\tau_{n, m}}\right),
$$

where $\tau^{0}$ is the longest CRD time of any cavity mode, i.e., it must be obtained with "clean" mirrors. The weighting factors have been calculated as $c_{m, n}=$ $1-k \Delta \Gamma_{n, m}$, where the factor $k$ provides the "contrast" in the reflectivity map. The, somewhat arbitrary, factor $k=250$ in Fig. $2(\mathrm{C})$ ensures that there is an acceptable dynamic range in the reflectivity map. In the experiments below we found that the optical loss due to contamination is typically less than $\Delta \Gamma_{n, m}=200 \mathrm{ppm}$ and $k$ was selected to rescale the weighing factor to about $c_{m, n}=0.5$.

\section{Experimental Apparatus}

The CRD apparatus is shown in Fig. 3 . A laser diode with a central wavelength of $639.7^{-} \mathrm{nm}(\sim 12 \mathrm{~mW}$, WA-1500, EXFO) was coupled into the cavity, which had an effective length of $60 \mathrm{~cm}$ and aperture of $\varnothing 20 \mathrm{~mm}$. The injection current of the laser was controlled by an integrated circuit (WLD3243, Wavelength Inc.) and could be switched off within about 


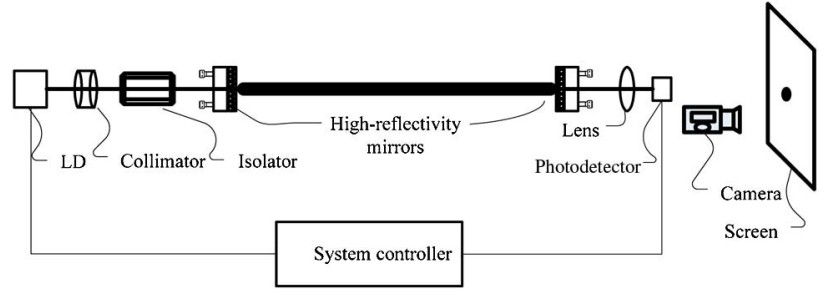

Fig. 3. Sketch of the experimental CRD apparatus.

$80 \mathrm{~ns}$. The collimated laser beam with a diameter of $\sim 3 \mathrm{~mm}$ was directed into a low-loss optical cavity. To avoid retroreflection into the laser diode, an optical isolator (IO-3D-633-VLP, OFR) was inserted into the optical path. The cavity was composed of two $\varnothing 25 \mathrm{~mm}$ supermirrors, which were made in-house. The mirrors were mounted on two-dimensional mechanical adjusters. The mirrors were all manufactured in the same batch. On their superpolished surface with the roughness of $\sim 1 \AA$ (RMS) (AFM3100, Veeco) multilayer dielectric coatings were deposited by ultralow loss ion beam sputtering. The central reflectivity wavelength and the bandwidth of the mirrors was determined to be $\sim 633 \mathrm{~nm}$ and $\sim 120 \mathrm{~nm}$, respectively, using a spectrometer (Lambda 950, PerkinElmer). To collect the light transmitted from the optical cavity, a Ø20 mm lens with $30 \mathrm{~mm}$ focal length was placed before the high-speed photodetector (APD110A, Thorlabs). As shown below, the average reflectivity was determined from ringdown time measurement. From the measured cavity loss of about $\Gamma=150 \mathrm{ppm}$ we calculate the average reflectivity as $R=1-\Gamma=99.985 \%$.

Unlike CRD spectrometers with high spectral resolution, our experimental apparatus does not require cavity length scanning or a laser wavelength fine-tuning mechanism, since the laser is always in resonance with several longitudinal cavity modes, because its wide spectral linewidth $(\sim 6 \mathrm{GHz}$, WA1500 and WA-650, EXFO) is much larger than the free spectral range of the optical cavity $(\sim 250 \mathrm{MHz})$. The output signal of the detector was connected to the A/D converter (CS320A, Cleverscope). To obtain the decay signal with high signal-to-noise ratio, the system controller set a threshold voltage to select the ringdown transient. Once the amplitude of the resonance signal transmitted from the optical cavity exceeded this threshold, the decay signal was captured and converted by the A/D converter. After fitting the data in a microcomputer, which is also included in the system controller, the cavity loss was determined. To record the ringdown waveforms of different transverse modes, the alignment of the optical cavity was adjusted using two-dimensional mechanical mirror adjustments, and the incident angle and position of the laser were changed correspondingly.

The bandwidth of the detector was about $50 \mathrm{MHz}$ and we cannot observe the transverse longitudinal mode beating at about $250 \mathrm{MHz}$. Beating between transverse modes was occasionally observed at
Table 1. Ringdown Times and Optical Loss Associated with Seven Different Transverse Cavity Modes for the Clean Cavity. ${ }^{a}$

\begin{tabular}{cc}
\hline$\tau / \mu \mathrm{s}$ & $\Gamma / \mathrm{ppm}$ \\
\hline $26.3(2)$ & $152.4(1.2)$ \\
$28.5(9)$ & $139.8(4.3)$ \\
$28.1(7)$ & $142.6(3.7)$ \\
$26.64(5)$ & $150.1(0.33)$ \\
27.1 & 147.5 \\
28.3 & 141.1 \\
25.1 & 159.6 \\
\hline
\end{tabular}

${ }^{a}$ For the first four modes three measurements were averaged and the respective standard deviation is given in brackets. The average ringdown time is 27.2(1.2) $\mu \mathrm{s}$ and the average loss is 147(8) ppm.

frequencies around $3 \mathrm{MHz}$, but those measurements were discarded.

\section{Experiments and Discussion}

The performance of the CRD apparatus was tested first using the $\mathrm{TEM}_{00}$ cavity mode. To protect the high-reflectivity mirrors from air-borne contamination, the entire apparatus was placed under an air filter and clean air flowed through the apparatus during the measurement. As described above, decay transients were captured and analyzed by fitting to an exponential decay function. For the mode shown in Fig. 4 the decay time and cavity loss were determined as $28.46 \mu \mathrm{s}$ and $139.8 \mathrm{ppm}$, respectively. For four transverse cavity modes three repeated measurements were used to evaluate the stability of the apparatus, and it was found that the standard deviations of these measurements were between 0.3

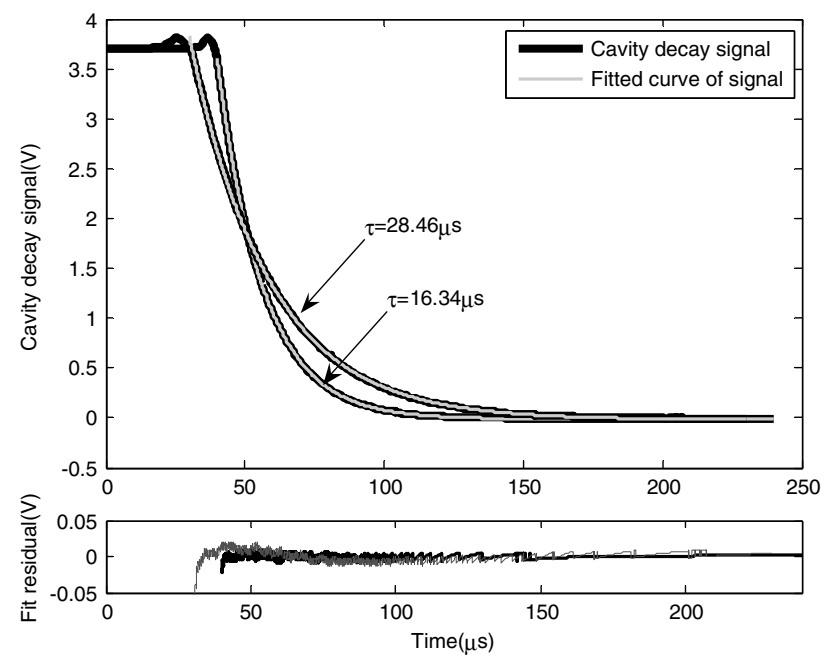

Fig. 4. Cavity decay signal of the second mode in Table 1 and its fit to a single exponential function giving $\tau_{0}=28.46 \mu \mathrm{s}$ and $\Gamma=$ $139.8 \mathrm{ppm}$ for one of the three measurements. After contamination of the mirrors a shorter ringdown time $\tau_{0}=16.34 \mu \mathrm{s}$ and $\Gamma=$ $244.4 \mathrm{ppm}$ is obtained from the transient of the highlighted mode in Fig. 6. 
and $1.0 \mathrm{ppm}$ (Table 1) and the average ringdown times were within $13 \mathrm{ppm}$ from one another. The ringdown measurements were then performed for three more transverse modes. The average optical loss associated with these modes was $147.3 \pm$ $7.8 \mathrm{ppm}$. Together these measurements indicate that all modes that were sampled experienced a similar optical loss. Since the experimentally observed modes are almost always linear combinations of the Hermite-Gaussian basis functions an assignment of the modes is complicated and was not attempted.

Importantly, we do not observe evidence of mode beating in the residual of Fig. 4B. Longitudinal mode beating is expected to be present on a time scale that cannot be resolved by our detection system (see Section 3 above), and transverse mode beating can be suppressed by careful alignment of the laser with respect to the cavity axis. While alignment cannot ensure that only a single transverse mode is excited, the residuals indicate that mode beating does not influence the accuracy of the ringdown time measurement.

Subsequently, the air filter was stopped and the cavity mirrors were exposed to ambient laboratory air for several hours. In this case, the reflectivity of the coatings deteriorated because the two mirror surfaces had adsorbed small dust particles floating in the air. We expected that both mirrors were contaminated to a similar extent. In Fig. 4, we show a representative example of the decay of a transverse mode obtained with contaminated mirrors. It is apparent that this decay curve can also be described with a single-exponential function, indicating that the existence of coating inhomogeneities does not distort the single exponential decay character of the ringdown signal. To identify the location of contamination on the mirrors quantitatively, 15 different transverse modes of the optical cavity were excited and their intensity distribution was captured together with their respective ringdown times (Fig. 5). The optical loss was determined for each mode in two to three measurements and the averages are included in the figure. The average optical loss of all modes has increased to $153.5 \pm 13.3 \mathrm{ppm}$. The large standard deviation indicates that the spread of the optical loss has also increased when different transverse modes are compared.

To determine the reflectivity map, $R^{\prime}(x, y)$, all 15 images were converted using ImageJ software (version 1.43, W. Rasband, National Institutes of Health, USA) into grayscale images, rescaled to full 16-bit range and divided by 15 . The images now all have roughly the same average intensity, i.e., they were normalized with respect to each other. A simple sum of the normalized images produces Fig. 7(A).

Next, we generate a similar image using the same normalized mode patterns but multiply each image with a factor, $c_{n, m}$, that was derived from the change in the modes' optical loss due to contamination, $\Delta \Gamma$ [Eq. (9)]. We calculate the optical loss due to mirror

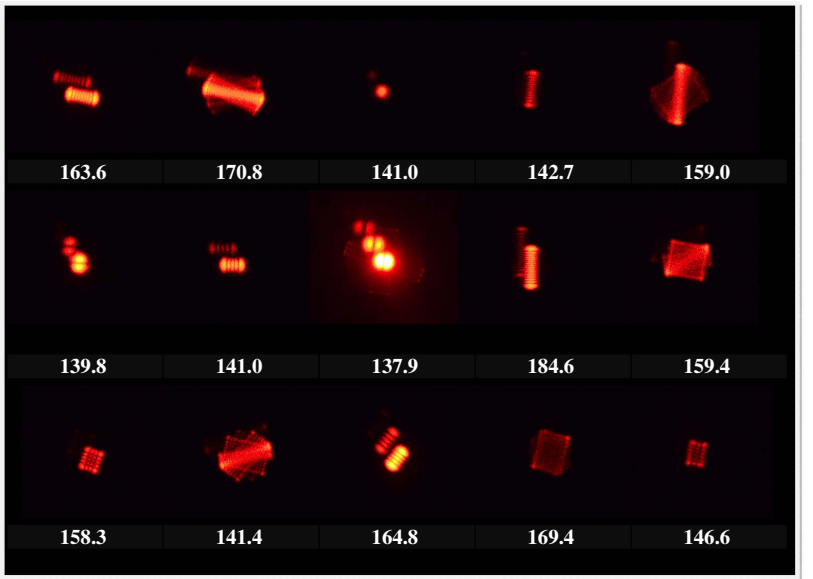

Fig. 5. Photographs of 15 different transverse cavity modes with respective optical loss (in ppm) after exposure to ambient air.

contamination by subtracting the lowest optical loss observed for a mode of the "clean" cavity, $\Gamma_{0}=$ $139.8 \mathrm{ppm}$. The 15 normalized images were multiplied with scaling factors that were calculated as $c=$ $(1-10000 \Delta \Gamma)$ and were between 0.55 and 1.00. The 15 weighted images are then added to produce a composite image [Fig. 7(B)]. As in Fig. 2 the ratio of the sums of the normalized images and of the weighted images provides the spatial distribution of the reflectivity [Fig. $7(\mathrm{C})]$. The ratio of two images produces a lot of noise in the region where both images are dark. We therefore also show the difference between the sum of the normalized images and the sum of the weighted images in Fig 7(D):

$$
R^{\prime \prime}(x, y)=\bar{I}(x, y)-\frac{\sum_{n, m=0}^{N, M} c_{m, n} I_{m, n}(x, y)}{(N+1)(M+1)} .
$$

In Figs. 7(C) and 7(D) we observe the largest decrease in reflectivity due to contamination in a wide spatial region near the center of the mirror. The composite image in Fig. $\underline{7(\mathrm{D})}$ provides a larger weight

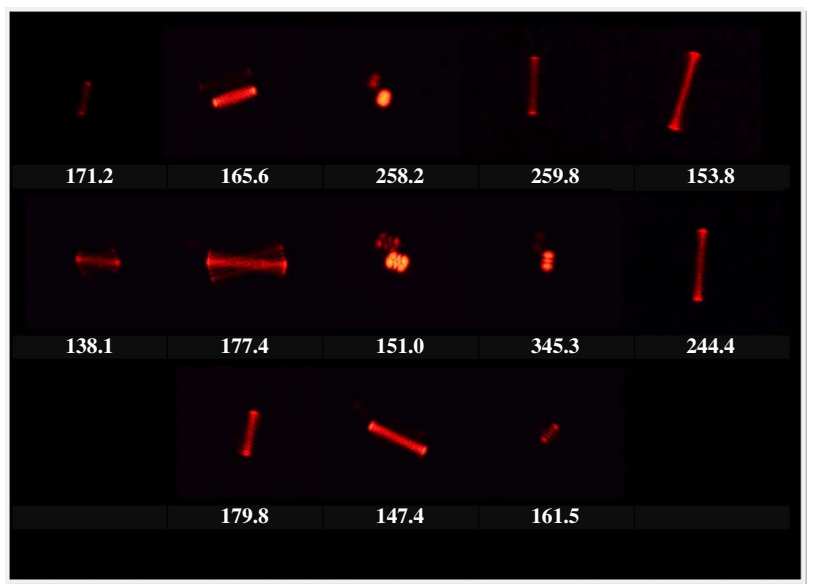

Fig. 6. Photographs of 13 different transverse cavity modes with respective optical loss (in ppm) after a spot was painted on one of the mirrors. 

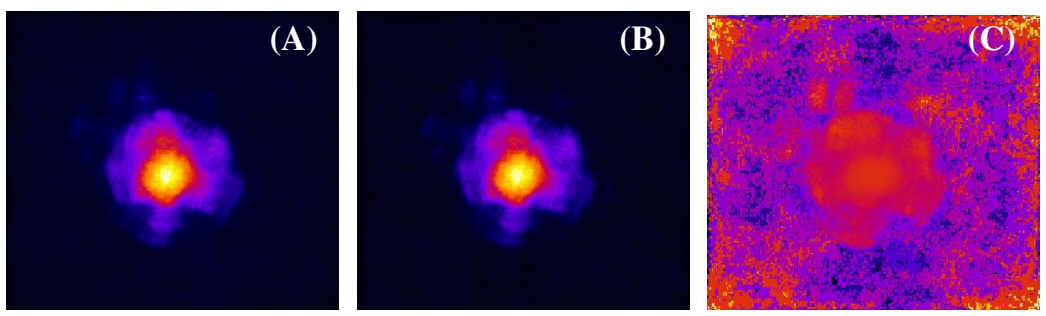

(D)
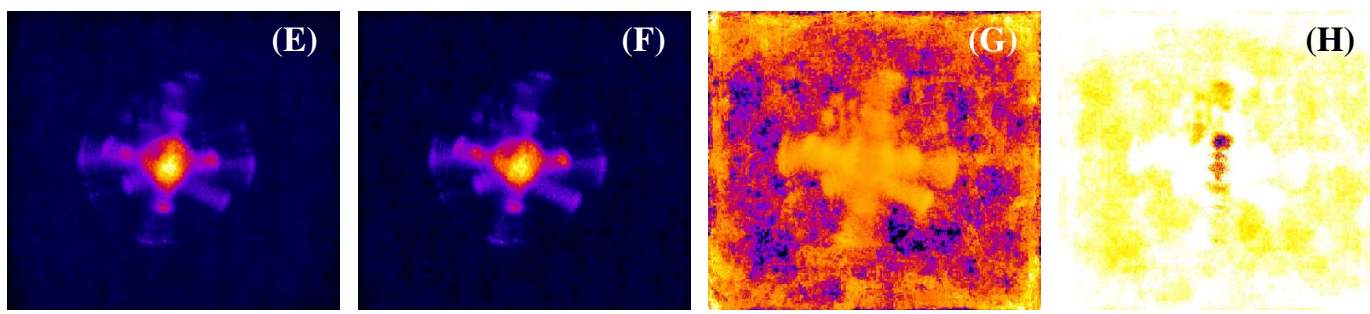

Fig. 7. Composite image obtained by adding normalized photographs of all transverse modes. (A) Sum of all modes shown in Fig. $\underline{5}$ after background subtraction and normalizing their intensity. (B) Sum of the same modes after weighting each normalized image by a factor dependent on the associated optical loss. (C) Ratio of (A) and (B). (D) Difference between (A) and (B). (E) Sum of all modes shown in Fig. 6 after background subtraction and normalizing their intensity. (F) Sum of the same modes after weighting each normalized image by a factor dependent on the associated optical loss. (G) Ratio of (E) and (F). (H) Difference between (E) and (F).

near regions of higher intensity and is more meaningful. Clearly, the reflectivity distribution in both images is not as distinct as the simulated distribution of Fig. 2, as may be expected. Of course, without additional measurements it is not possible to identify which of the two mirrors contributes to the optical loss of a particular cavity mode.

The experiment was then repeated using a clean cavity in which one of the mirrors had a small dot $(\varnothing 200-400 \mu \mathrm{m})$ painted near the center of the cavity axis. A different set of 13 transverse cavity modes was photographed and their optical loss was calculated from their respective average ringdown times [Fig. 6]. As above, the normalized composite image was obtained [Fig. 7(E)] and the weighted composite image was generated [Fig. $7(\mathrm{~F})]$ as above but with $k=1000$. The ratio between the two images [Fig. $7(\mathrm{G})$ ] does not give a very distinct reflectivity map, but the difference between the two images [Fig. $7(\mathrm{H})]$ now clearly shows a small region that is responsible for the cavity loss. The dot painted on the mirror only affects those cavity modes that are sampling this region of the mirror. The optical loss of these modes was as high as $345 \mathrm{ppm}$ while other cavity modes that do not sample this region of the detector have an optical loss that is comparable with that of a clean cavity (139.8 ppm).

The spatial resolution in this experiment is ultimately given by the theoretical limit of about $\delta x \delta y=4 w^{2} N^{-1 / 2} M^{-1 / 2}$, but is here also limited by the resolution of the images taken with the camera. It is estimated to be in the range of several hundreds of micrometers and comparable to the size of the dot. A larger number of images would have also served to provide a better spatial resolution.

More accurate results would have been obtained, had it been possible to obtain the ringdown time of every mode before and after contamination, but in practice this is difficult, since the mode pattern cannot be exactly reproduced in two independent experimental runs. It was therefore necessary to determine a common $\tau_{0}$ and $\Gamma_{0}$ for all modes, limiting the accuracy of the measurement.

Many of the excited cavity modes were not Hermite-Gaussian modes, but rather a linear combination of a number of $\mathrm{TEM}_{m, n}$ functions. This did not seem to affect the results negatively but instead helped break the symmetry of the composite image and thereby aided in locating the "defect site" unambiguously. The model presented above also holds when a number of TEM modes are excited simultaneously as long as their ringdown times are close to each other.

\section{Conclusion}

In conclusion, the ringdown time measurements together with a measured intensity distribution on the mirror surfaces can give an indication where a coating defect may be located on the mirrors. Even if the precise location of the contamination is not of great concern, such measurements can be used to assure that the mirror is coated homogeneously. A large extent of inhomogeneity results in a large variation in ringdown times. The accuracy with which the defect can be located is directly related to the number of basis functions, i.e., the number of transverse modes in the composite image. It was apparent that the existence of coating inhomogeneities does not distort the decay signal, but only introduces optical loss differences between various modes of the ringdown cavity [23].

The work was sponsored by the National Natural Science Foundation of China (61205157). HPL acknowledges financial support by the Natural Sciences and Engineering Research Council of 
Canada. The authors gratefully acknowledge the insightful comments by both referees.

\section{References}

1. Y. He and B. J. Orr, "Detection of trace gases by rapidly-swept continuous-wave cavity ringdown spectroscopy: pushing the limits of sensitivity,” Appl. Phys. B 85, 355-364 (2006).

2. D. Romanini and K. K. Lehmann, "Ring-down cavity absorption spectroscopy of the very weak HCN overtone bands with six, seven, and eight stretching quanta," J. Chem. Phys. 99, 6287-6301 (1993).

3. J. M. Herbelin, J. A. McKay, M. A. Kwok, R. H. Ueunten, D. S. Urevig, D. J. Spencer, and D. J. Benard, "Sensitive measurement of photon lifetime and true reflectances in an optical cavity by a phase-shift method," Appl. Opt. 19, 144-147 (1980).

4. D. Z. Anderson, J. C. Frisch, and C. S. Masser, "Mirror reflectometer based on optical cavity decay time," Appl. Opt. 23, 1238-1245 (1984).

5. Z. Q. Tan, X. Long, K. Yang, and S. Wu, "Spectral ripple effect in continuous-wave fold-type cavity ring down spectroscopy," J. Opt. Soc. Am. B 27, 2727-2730 (2010).

6. Y. Gong, Y. Han, and B. Li, "Effect of threshold value on high reflectivity measurement with optical feedback cavity ringdown technique," Proc. SPIE 7283, 72830U (2009).

7. G. Berden and R. Engeln, eds., Cavity Ring-Down Spectroscopy: Techniques and Applications (Wiley-Blackwell, 2009).

8. G. Berden, R. Peeters, and G. Meijer, "Cavity ring-down spectroscopy: Experimental schemes and applications," Int. Rev. Phys. Chem. 19, 565-607 (2000).

9. M. Mazurenka, A. J. Orr-Ewing, R. Peverall, and G. A. D. Ritchie, "Cavity ring-down and cavity enhanced spectroscopy using diode lasers," Ann. Rep. Prog. Chem., Sect. C 101, 100-142 (2005).

10. C. Vallance, "Innovations in cavity ringdown spectroscopy," New J. Chem. 29, 867-874 (2005).

11. C. C. Harb, T. K. Boyson, A. G. Kallapur, I. R. Petersen, M. E. Calzada, T. G. Spence, K. P. Kirkbride, and D. S. Moore, "Pulsed quantum cascade laser-based CRDS substance detection: real-time detection of TNT," Opt. Express 20, 15489-15502 (2012).

12. K. K. Lehmann and D. Romanini, "The superposition principle and cavity ring-down spectroscopy," J. Chem. Phys. 105, 10263-10277 (1996).

13. J. T. Hodges and D. Lisak, "Frequency-stabilized cavity ring-down spectrometer for high-sensitivity measurements of water vapor concentration,” Appl. Phys. B 85, 375-382 (2006).

14. D. Romanini, A. A. Kachanov, N. Sadeghi, and F. Stoeckel, "CW cavity ring down spectroscopy," Chem. Phys. Lett. 264, 316-322 (1997).

15. B. A. Paldus, C. C. Harb, T. G. Spence, B. Wilke, J. Xie, J. S. Harris, and R. N. Zare, "Cavity-locked ring-down spectroscopy,” J. Appl. Phys. 83, 3991-3997 (1998).

16. A. Cygan, D. Lisak, P. Maslowski, K. Bielska, S. Wojtewicz, J. Domyslawska, R. S. Trawinski, R. Ciurylo, H. Abe, and J. T. Hodges, "Pound-Drever-Hall-locked, frequency-stabilized cavity ring-down spectrometer," Rev. Sci. Instrum. 82, 063107 (2011).

17. T. Klaassen, J. de Jong, M. van Exter, and J. P. Woerdman, "Transverse mode coupling in an optical resonator," Opt. Lett. 30, 1959-1961 (2005)

18. H. F. Huang and K. K. Lehmann, "Noise in cavity ring-down spectroscopy caused by transverse mode coupling," Opt. Express 15, 8745-8759 (2007).

19. B. A. Paldus and A. A. Kachanov, "An historical overview of cavity-enhanced methods," Can. J. Phys. 83, 975-999 (2005).

20. W. T. Silfvast, Laser Fundamentals (Cambridge University, 2008).

21. H. Kogelnik and T. Li, "Laser beams and resonators," in Proceedings of the Institute of Electrical and Electronics Engineers (1966), Vol. 54, p. 1312.

22. A. E. Siegman, Lasers (including the author's list of corrections), 1st ed. (University Science Books, 1986).

23. Z. Q. Tan and X. W. Long, "A developed optical-feedback cavity ring-down spectrometer and its application," Appl. Spectrosc. 66, 492-495 (2012). 